\title{
The potential adjunctive benefit of adding metformin to standard treatment in inoperable cancer patients: a meta-analysis of randomized controlled trials
}

\author{
Zhonghua Wü, Bicheng Qu", Xuanzhang Huang, Yongxi Song, Peng Gao, Jinxin Shi, Cen Zhou, \\ Zhenning Wang
}

Department of Surgical Oncology and General Surgery, Key Laboratory of Precision Diagnosis and Treatment of Gastrointestinal Tumors, Ministry of Education, The First Affiliated Hospital of China Medical University, Shenyang, China

Contributions: (I) Conception and design: Z Wu, Z Wang; (II) Administrative support: Y Song; (III) Provision of study materials or patients: B Qu, X Huang; (IV) Collection and assembly of data: Z Wu, B Qu, C Zhou, P Gao; (V) Data analysis and interpretation: Z Wu, P Gao, J Shi; (VI) Manuscript writing: All authors; (VII) Final approval of manuscript: All authors.

\#These authors contributed equally to this work.

Correspondence to: Prof Zhenning Wang. Department of Surgical Oncology and General Surgery, Key Laboratory of Precision Diagnosis and Treatment of Gastrointestinal Tumors, Ministry of Education, The First Affiliated Hospital of China Medical University, 155 North Nanjing Street, Heping District, Shenyang, 110001, China. Email: josieon826@sina.cn.

Background: Recently, there have been several randomized clinical trials (RCTs) conducted to evaluate the efficacy and safety of metformin plus standard treatment in inoperable cancer patients. Our meta-analysis aimed to assess the efficacy of metformin in combination with standard treatment in inoperable cancer patients.

Methods: PubMed and Embase databases were systematically searched for relevant RCTs investigating the efficacy of adding metformin to standard treatment for cancer patients. The pooled relative risk (RR) for tumor response and safety was calculated to assess the efficacy of combining metformin with standard treatment. Meta-analysis was subsequently performed to pool the hazard ratio (HR) for overall survival (OS) and progression-free survival (PFS).

Results: Ten RCTs comprising 1033 patients were included in our current meta-analysis. In patients with breast cancer, results of meta-analysis showed that the addition of metformin to standard treatment was beneficial to objective response rate (ORR) with 30.3\% (33/109) in the metformin plus standard treatment group and $16.1 \%$ (18/112) in the placebo group (RR 1.92, 95\% CI: 1.19-3.10, P=0.008). OS and PFS were not significantly improved in patients who received metformin plus standard treatment compared with those who received placebo plus standard treatment (OS: HR 1.02, 95\% CI: 0.71-1.46, P=0.916; PFS: HR 1.14, 95\% CI: 0.86-1.50, P=0.366). For lung cancer patients, meta-analysis results showed adding metformin to standard treatment could benefit ORR (metformin $65.3 \%$ vs. placebo 54.6\%, RR 1.22, 95\% CI: $1.03-1.43$, $\mathrm{P}=0.018$ ) with no significant survival benefit in the metformin group. For patients with pancreatic cancer, the pooled ORR was $17.6 \%(16 / 91)$ in metformin plus standard treatment group and $20 \%(18 / 90)$ in the placebo group, indicating metformin did not benefit ORR (RR 0.85, 95\% CI: 0.49-1.49, P=0.576). Besides, the addition of metformin to standard treatment did not increase the incidence rate of adverse effects.

Conclusions: Our results indicated that addition of metformin to standard treatment was beneficial to ORR in inoperable breast or lung cancer patients without increasing the incidence of adverse effects. However, adding metformin to standard treatment could not benefit OS and PFS.

Keywords: Metformin; cancer; meta-analysis; overall survival (OS); progression-free survival (PFS)

Submitted Jun 03, 2020. Accepted for publication Sep 04, 2020.

doi: $10.21037 / \mathrm{atm}-20-4441$

View this article at: http://dx.doi.org/10.21037/atm-20-4441 


\section{Introduction}

The global cancer incidence and mortality rates are increasing rapidly, with an estimated 18.1 million new cases and 9.6 million cancer-related deaths reported in 2018 worldwide (1). The Global Burden of Disease Study (2) has reported that cancer is a severe obstacle in improving the life expectancy of humans worldwide. Although many advancements have been made in the treatment of cancer, the survival rate of different types of cancer has not increased proportionally. Currently, the research on chemotherapy and discovery of novel monoclonal antibodies have revolutionized cancer therapy. However, the therapeutic role of conventional non-anti-cancer drug in combination with chemotherapy is not clear.

In recent years, several conventional non-anti-cancer drugs, such as non-steroid anti-inflammatory drugs (NSAIDs), statin, metformin, have gained much attention for their anti-cancer properties. Several epidemiological studies (3-5) have reported significant survival benefits in cancer patients using these drugs. Other studies (6-9) have revealed that these drugs exert anti-cancer effects both in vitro and in vivo, and could work synergistically with chemotherapeutic drugs to inhibit tumor growth. However, few clinical trials have investigated the adjuvant therapeutic efficacy of these drugs in cancer patients. Whether adding these non-anti-cancer drugs to standard clinical treatments offers adjunctive benefit to cancer patients is still unclear.

As a conventional anti-diabetic drug, metformin has been used in the treatment of type II diabetes mellitus for over 30 years (10). Several experimental studies have reported that metformin has anti-cancer effects in lung cancer (7), pancreatic cancer (11) and gastric cancer (12) etc. In addition, population-based studies have indicated that metformin use correlated with a reduced incidence rate of various cancers (13-16). Meanwhile, many primary researches and meta-analyses reported that metformin use was associated with an improved survival outcome in pancreatic cancer (17-19), lung cancer (20,21), breast cancer $(22,23)$, or colorectal cancer (4), etc. Although several RCTs have been conducted to assess the efficacy and safety of adding metformin to standard treatment in inoperable cancer patients, the findings are inconsistent.

As RCTs are the golden standard for evaluating the efficacy of various interventions, we conducted this metaanalysis based on recently published RCTs to assess the efficacy of metformin combined with standard treatment in inoperable cancer patients. We present the following article in accordance with the PRIMSA reporting checklist (Available at http://dx.doi.org/10.21037/atm-20-4441).

\section{Methods}

This study was approved by the institutional Ethics Committee of the First Affiliated Hospital of China Medical University (IRB approval number:AF-SOP-07-1.1-01). The informed consent was waived because this study was a metaanalysis of previously published RCTs and did not involve any processing of individual patient data.

\section{Literature search}

The PubMed and Embase databases were systematically searched in September 2019 for relevant RCTs that examined the efficacy of metformin combined with standard treatment for cancer patients. The search strategy was performed corresponding to the following terms: "dimethylbiguanidine", "dimethylguanylguanidine", "glucophage", "metformin", "melbine", "cancer", "neoplasm", "tumor", "malignant*", "randomized trial", "randomized trial", "randomized study", and "randomized study". The references of relevant studies were also carefully reviewed to find several potentially studies for inclusion. Conference abstracts from the annual meetings of the American Society of Clinical Oncology (ASCO), the European Society for Medical Oncology (ESMO), and Chinese Society of Clinical Oncology (CSCO) were also reviewed to identify relevant unpublished data.

\section{Identification of eligible studies}

Two investigators (Z.H.W and B.C.Q) independently reviewed all the retrieved studies to identify the eligible studies. The goal of current study was to evaluate whether adding metformin to standard treatment confer therapeutic efficacy in inoperative cancer patients. Based on this objective, the following inclusion criteria were used to include eligible studies for meta-analysis: (I) Patients: patients were diagnosed with advanced or metastatic cancer and treated with systematic standard chemotherapy; (II) Intervention: Standard treatment with metformin or placebo were randomized to cancer patients; (III) Comparison: the control group received standard treatment with placebo; (IV) Outcomes: the primary outcomes of current study were the objective response rate (ORR), progression-free 


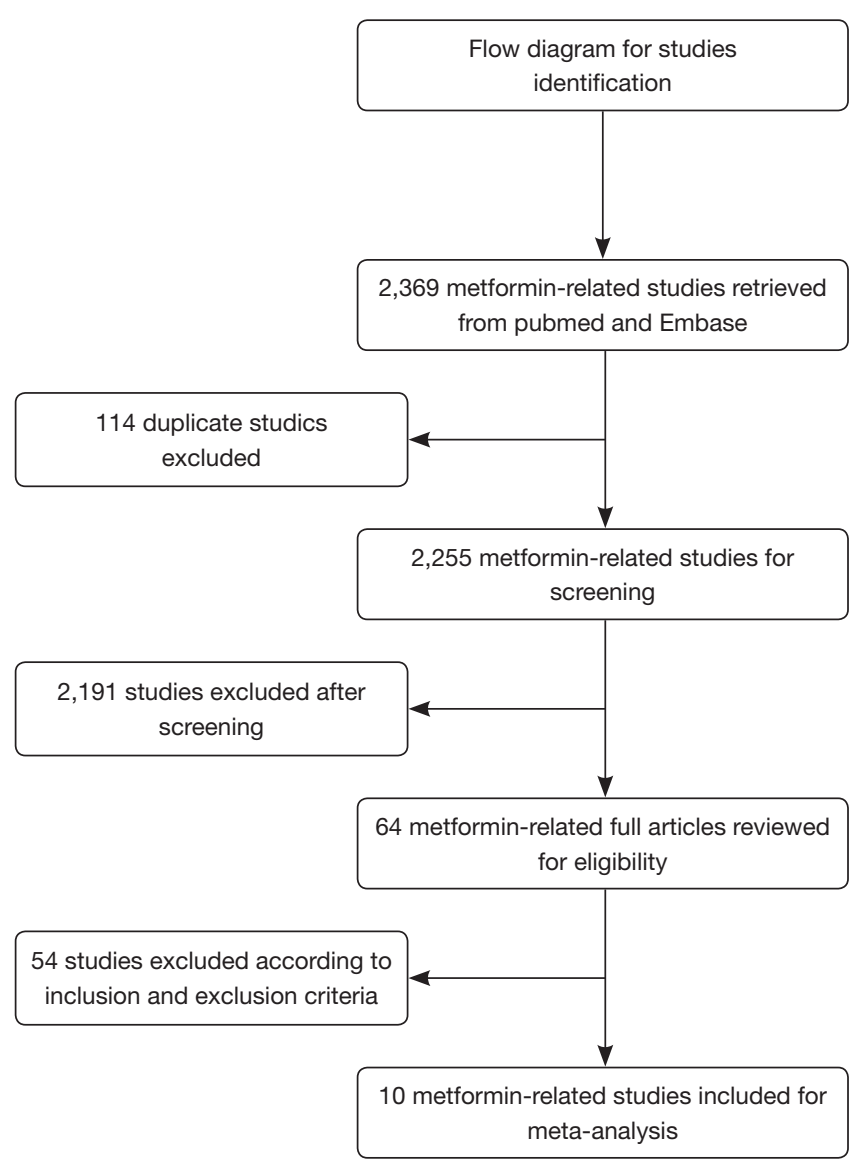

Figure 1 The flow chart of literature search and study selection.

survival (PFS), overall survival (OS), and drug safety; the outcome measures and $95 \%$ CIs were reported in studies or could be calculated by extracting relevant data provided by the studies; and (V) study design: studies were phase II or III RCTs.

Studies were excluded if: (I) animal, in vitro studies or non-RCTs were conducted to evaluate the efficacy of metformin; (II) patients included in studies were diagnosed with type 1 or 2 diabetes; (III) patients received anti-cancer treatment before enrolling in studies; and (IV) the relevant outcomes data could not be obtained in studies.

\section{Data extraction}

The relevant data from eligible RCTs were extracted by two investigators (Z.H.W and B.C.Q) independently. Information on the first author, year of publication, continent, study design, cancer type and stage, standard chemotherapy regimen, type of conventional drug, dose of drug used and sample size was collected. Any discrepancy between the two investigators was resolved by discussion according to the Quality of Reporting of Meta-Analyses Guidelines (24).

\section{Statistical analysis}

The pooled relative risks (RRs) and $95 \%$ CIs were calculated to assess the effects of metformin combined with standard treatment on the ORR and adverse effects (AEs) rate for grades $\geq 3$. For metformin combined with standard treatment, an $\mathrm{RR}<1$ indicated a lower ORR and AEs rate, whereas an $\mathrm{RR}>1$ indicated a higher ORR and AEs rate. The hazard ratios (HRs) and $95 \% \mathrm{CI}$ : from individual trials were extracted and pooled to evaluate the effects of metformin plus standard treatment versus placebo plus standard treatment on PFS and OS. For metformin combined with standard treatment, an $\mathrm{HR}<1.0$ indicated a longer PFS and OS, whereas an HR $>1.0$ indicated a shorter PFS and OS.

Statistical heterogeneity across RCTs was assessed through the Cochrane $\mathrm{Q}$ test and $\mathrm{I}^{2}$ statistic, and $\mathrm{I}^{2}<50 \%$ and/or $\mathrm{P}>0.05$ indicated there was no significant heterogeneity (25). A fixed-effects model was selected for analysis if there was no evident heterogeneity among studies; otherwise, a random-effects model was used. Subgroup analyses were carried out to evaluate the effects of continent, cancer type, and metformin dosage on the efficacy of combining either drug with standard treatment for cancer patients. Publication bias was assessed using Begg's and Egger's tests (26,27).

All statistical analyses were performed using Stata software (version 12.0, Stata Corporation, College Station, TX, USA). Pooled HRs and RRs were recognized as statistically significant if the $95 \%$ CI: did not include 1.0 and the $\mathrm{P}$ value was $<0.05$ (two-sided).

\section{Results}

\section{Study selection and characteristics}

The flow diagram of the study selection process is shown in Figure 1. A total of 2369 metformin-related studies were retrieved from PubMed and Embase databases, of which 114 duplicate studies were excluded. Among the remaining 2,255 studies, 2,191 studies were excluded after reviewing the titles and abstracts. The remaining 64 studies were then 
screened by carefully reviewing the full texts. Finally, 10 studies (28-37) were included in our study to investigate the efficacy of metformin plus standard treatment versus placebo plus standard treatment.

The included studies were published between 2014 and 2019 in the United States of America, Italy, Netherlands, Canada, Mexico, Egypt, Korea, and China. There were 1033 patients included in our current meta-analysis. The main characteristics of these RCTs are summarized in Table 1 and quality assessments of these RCTs are shown in Table S1.

\section{Efficacy of metformin in breast cancer}

There are three RCTs including 226 participants investigating the efficacy of adding metformin to standard treatment in breast cancer. These RCTs reported the ORR, OS and PFS data. The pooled ORR was 30.3\% (33/109) in the metformin combined with treatment group and $16.1 \%$ $(18 / 112)$ in the placebo combined with standard treatment group, showing that the addition of metformin to standard treatment was beneficial to the ORR (RR 1.92, 95\% CI: $1.19-3.10, \mathrm{P}=0.008)$ with no significant heterogeneity $\left(\mathrm{I}^{2}=26.7 \%, \mathrm{P}_{\text {het }}=0.255\right)$ (Figure $\left.2 A\right)$. However, the results of meta-analysis showed that OS and PFS were not significantly improved in patients who received metformin plus standard treatment compared with those who received placebo plus standard treatment without significant heterogeneity (OS: HR 1.02, 95\% CI: 0.71-1.46, P=0.916, $\mathrm{I}^{2}=23.8 \%, \mathrm{P}_{\mathrm{het}}=0.269$; PFS: HR 1.14, 95\% CI: 0.86-1.50, $\mathrm{P}=0.366, \mathrm{I}^{2}=0 \%, \mathrm{P}_{\text {het }}=0.945$ ) (Figure $2 B, C$ ).

\section{Efficacy of metformin in lung cancer}

Four RCTs (418 patients) investigated the efficacy of adding metformin to standard treatment in lung cancer. ORR and OS were reported in these four RCTs, and PFS was assessed in three of these RCTs. Meta-analysis results showed adding metformin to standard treatment could benefit ORR (metformin $65.3 \%$ vs. placebo $54.6 \%$, RR $1.22,95 \%$ CI: $1.03-1.43, \mathrm{P}=0.018$ ) with no significant heterogeneity $\left(\mathrm{I}^{2}=30.4 \%, \mathrm{P}_{\mathrm{het}}=0.230\right)$ (Figure $\left.3 A\right)$. The results showed the addition of metformin to standard treatment did not improve OS and PFS in lung cancer patients [OS: HR 0.88, 95\% CI: $0.65-1.19, \mathrm{P}=0.409, \mathrm{I}^{2}=49.1 \%, \mathrm{P}_{\mathrm{het}}=0.117$; PFS (random effect): HR 0.63, 95\% CI: $0.32-1.27, \mathrm{P}=0.197$, $\left.\mathrm{I}^{2}=77.5 \%, \mathrm{P}_{\text {het }}=0.012\right]($ Figure $3 B, C)$.

\section{Efficacy of metformin in pancreatic cancer}

There are two RCTs (181 patients) assessing the efficacy of metformin plus standard treatment in pancreatic cancer. The pooled ORR was $17.6 \%$ (16/91) in metformin plus standard treatment group and 20\% (18/90) in the placebo group, showing metformin did not benefit ORR (RR 0.85, 95\% CI: 0.49-1.49, $\mathrm{P}=0.576)$ without significant heterogeneity $\left(\mathrm{I}^{2}=0.0 \%, \mathrm{P}_{\text {het }}=0.709\right)$ (Figure $\left.4 A\right)$. Metaanalysis on OS and PFS showed adding metformin to standard treatment did not bring benefit to survival outcome (OS: HR 1.00, 95\% CI: 0.74-1.37, P=0.964, $\mathrm{I}^{2}=0 \%, \mathrm{P}_{\text {het }}=0.680$; PFS: HR 0.97, 95\% CI: 0.70-1.35, $\mathrm{P}=0.859, \mathrm{I}^{2}=49.0 \%, \mathrm{P}_{\text {het }}=0.161$ ) (Figure 4B,C).

\section{Safety of metformin}

Some commonly reported grade 3-4 AEs, including hematologic toxicities (neutropenia, febrile neutropenia, leucopenia, anemia, thrombocytopenia), digestive toxicities (nausea, vomiting, diarrhea) and other toxicities (fatigue, mucositis, infection, alopecia, anorexia, rash, hand-foot syndrome) were analyzed in our meta-analysis. The pooled analysis showed that AEs were generally similar between patients who received metformin combined with standard treatment and those who received placebo combined with standard treatment, except for a slightly higher incidence of diarrhea in the metformin plus standard chemotherapy group (8.0\% versus 4.2\%; RR 1.871, 95\% CI: $1.002-3.494$, $\mathrm{P}=0.049 ; \mathrm{I}^{2}=0 \%, \mathrm{P}_{\text {het }}=0.929$ ) (Table 2).

\section{Metformin dose}

We also examine the impact of metformin dose on the ORR and survival outcomes. Because of the limited number of studies for subgroup analysis in each cancer type, we pooled studies investigating these cancer types together and then performed subgroup analysis based on metformin doses. Subgroup analysis results showed both 1,000 and $500 \mathrm{mg}$ metformin daily could benefit the ORR when adding to standard treatment $(1,000 \mathrm{mg}$ daily: RR $1.28,95 \% \mathrm{CI}$ : $1.05-1.56, \mathrm{P}=0.015 ; \mathrm{I}^{2}=71.6 \%, \mathrm{P}_{\text {het }}=0.03 ; 500 \mathrm{mg}$ daily: RR 1.41, 95\% CI: $1.09-1.84, \mathrm{P}=0.010 ; \mathrm{I}^{2}=0 \%, \mathrm{P}_{\text {het }}=0.403$ ) (Figure 5). Subgroup analysis was also performed to assess the impact of metformin dose on OS and PFS. The results showed metformin doses have no significant relationships with OS (1,000 mg daily: HR 1.00, 95\% CI: 0.75-1.35, 


\begin{tabular}{|c|c|c|c|c|c|c|c|c|c|c|}
\hline 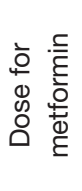 & 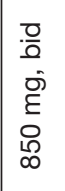 & $\begin{array}{l}\frac{0}{0} \\
\overline{0} \\
\xi \\
8 \\
8 \\
-\end{array}$ & $\begin{array}{l}0 \\
0 \\
0 \\
8 \\
8 \\
8 \\
8 \\
0\end{array}$ & $\begin{array}{l}\frac{0}{0} \\
\overline{0} \\
\dot{\varepsilon} \\
0 \\
0 \\
0\end{array}$ & $\begin{array}{l}\frac{0}{0} \\
\overline{0} \\
\frac{9}{8} \\
8 \\
0 \\
-\end{array}$ & 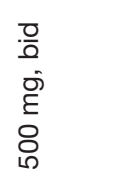 & $\begin{array}{l}\overline{0} \\
\overline{0} \\
\bar{g} \\
\bar{\varepsilon} \\
\dot{8} \\
0\end{array}$ & $\begin{array}{l}\text { 응 } \\
\overline{0} \\
\dot{E} \\
\stackrel{0}{0} \\
0\end{array}$ & $\begin{array}{l}\text { 음 } \\
\overline{0} \\
\xi \\
8 \\
8 \\
\text { i }\end{array}$ & $\begin{array}{l}\text { 음 } \\
\overline{8} \\
\xi \\
8 \\
8 \\
\text { ¿ }\end{array}$ \\
\hline 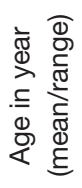 & 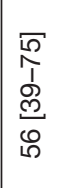 & $\begin{array}{l}\overline{8} \\
\frac{1}{1} \\
\frac{1}{0} \\
8\end{array}$ & 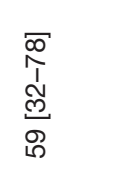 & 요 & $\begin{array}{l}\text { T } \\
\hat{1} \\
\hat{m}\end{array}$ & $\begin{array}{l}\bar{\infty} \\
\hat{1} \\
\infty \\
\infty \\
\hat{\infty}\end{array}$ & $\begin{array}{l}\bar{D} \\
\infty \\
1 \\
\infty \\
\end{array}$ & 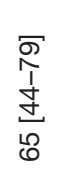 & 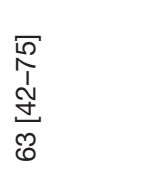 & $\frac{\Upsilon}{z}$ \\
\hline 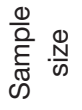 & P & $\stackrel{\stackrel{N}{\sim}}{\sim}$ & $\stackrel{\sim}{N}$ & 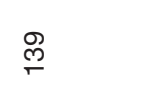 & $\stackrel{\llcorner}{N}$ & 8 & ஜ్లి & $\stackrel{\underset{\sim}{\sim}}{ }$ & 8 & $\stackrel{\infty}{\sim}$ \\
\hline $\begin{array}{l}\text { O } \\
\text { 异 } \\
\text { 焉 }\end{array}$ & $\tilde{\jmath}$ & ָ̃ & ָे & İ & $\overline{1}$ & $\bar{\jmath}$ & ָे & & $\frac{\mathfrak{r}}{z}$ & $\frac{\mathfrak{r}}{z}$ \\
\hline 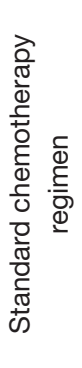 & 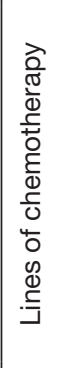 & 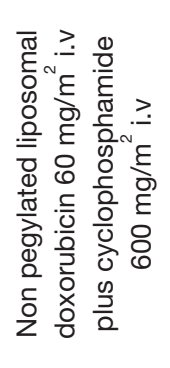 & 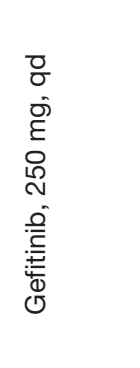 & 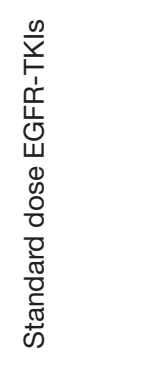 & 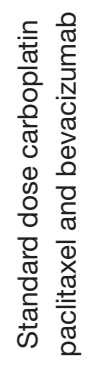 & 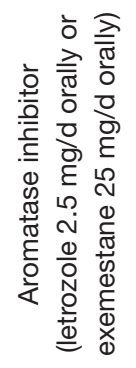 & 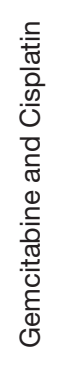 & 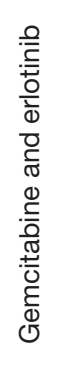 & 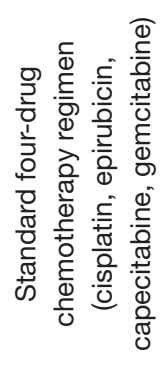 & 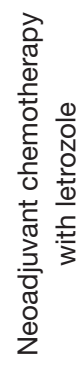 \\
\hline 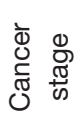 & $\geq$ & $\geq$ & 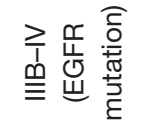 & 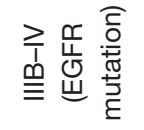 & $\begin{array}{l}\geq \\
\stackrel{1}{\equiv} \\
\equiv\end{array}$ & $\geq$ & $\geq$ & $\stackrel{\gtrless}{\equiv}$ & $\geq$ & $\stackrel{\bar{\equiv}}{\underline{I}}$ \\
\hline 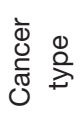 & O্口 & O্口 & $\begin{array}{l}0 \\
\mathcal{U} \\
\stackrel{W}{Z}\end{array}$ & 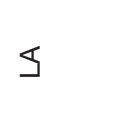 & $\begin{array}{l}0 \\
\text { O } \\
\text { O }\end{array}$ & O্口 & $\begin{array}{l}0 \\
0 \\
0 \\
Z\end{array}$ & O & O & O্ \\
\hline $\begin{array}{l}\text { Dे } \\
\text { 今ે } \\
0\end{array}$ & $\begin{array}{l}\frac{\pi}{0} \\
\frac{\pi}{\pi} \\
\frac{\pi}{\pi} \\
0\end{array}$ & 离 & 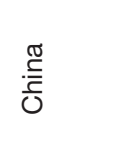 & $\begin{array}{l}\frac{0}{x} \\
\frac{0}{x}\end{array}$ & 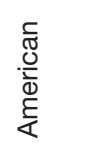 & 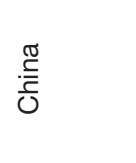 & 䓂 & 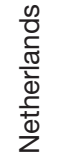 & $\underset{ \pm}{\stackrel{\vec{\pi}}{ \pm}}$ & $\begin{array}{l}\mathbb{\varpi} \\
\stackrel{\varpi}{0} \\
\check{0}\end{array}$ \\
\hline 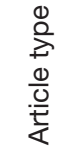 & 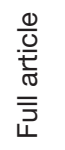 & 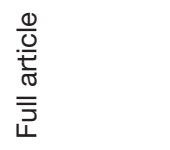 & 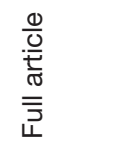 & 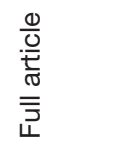 & 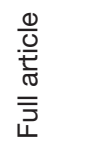 & 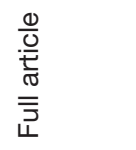 & 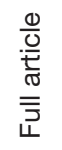 & 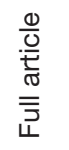 & 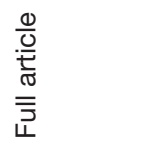 & 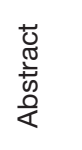 \\
\hline 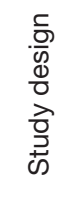 & 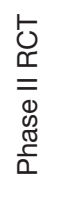 & 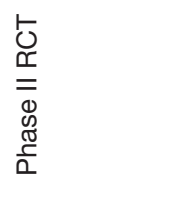 & 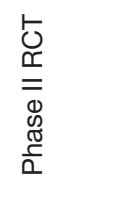 & 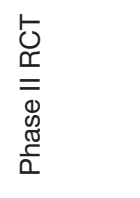 & 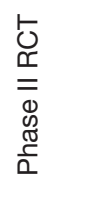 & 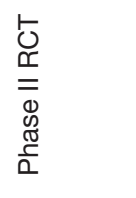 & 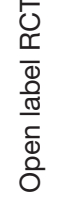 & 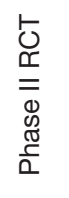 & 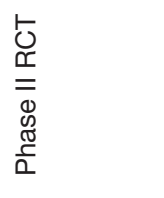 & 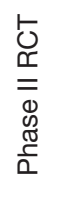 \\
\hline 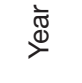 & $\stackrel{\circ}{\stackrel{\circ}{N}}$ & $\stackrel{\circ}{\stackrel{\circ}{2}}$ & $\stackrel{\circ}{\stackrel{\circ}{N}}$ & $\stackrel{0}{\stackrel{N}{N}}$ & $\stackrel{\infty}{\stackrel{\infty}{N}}$ & 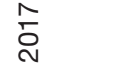 & $\stackrel{10}{\stackrel{2}{2}}$ & $\stackrel{10}{\stackrel{2}{2}}$ & $\stackrel{0}{\stackrel{0}{\sim}}$ & i \\
\hline $\begin{array}{l}\frac{1}{\circ} \\
\frac{1}{+} \\
\frac{1}{4}\end{array}$ & 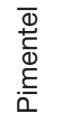 & $\begin{array}{l}\overline{\bar{\Sigma}} \\
\overline{\tilde{N}}\end{array}$ & ב & $\frac{\pi}{\frac{\pi}{0}}$ & 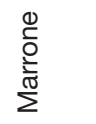 & $\begin{array}{l}\frac{8}{\pi} \\
\frac{\pi}{N}\end{array}$ & 离 & $\begin{array}{l}00 \\
\frac{0}{0} \\
\overline{0} \\
\underline{1}\end{array}$ & $\begin{array}{l}\overline{\bar{d}} \\
\widetilde{\varpi}\end{array}$ & $\xi \underline{\underline{\Sigma}}$ \\
\hline
\end{tabular}




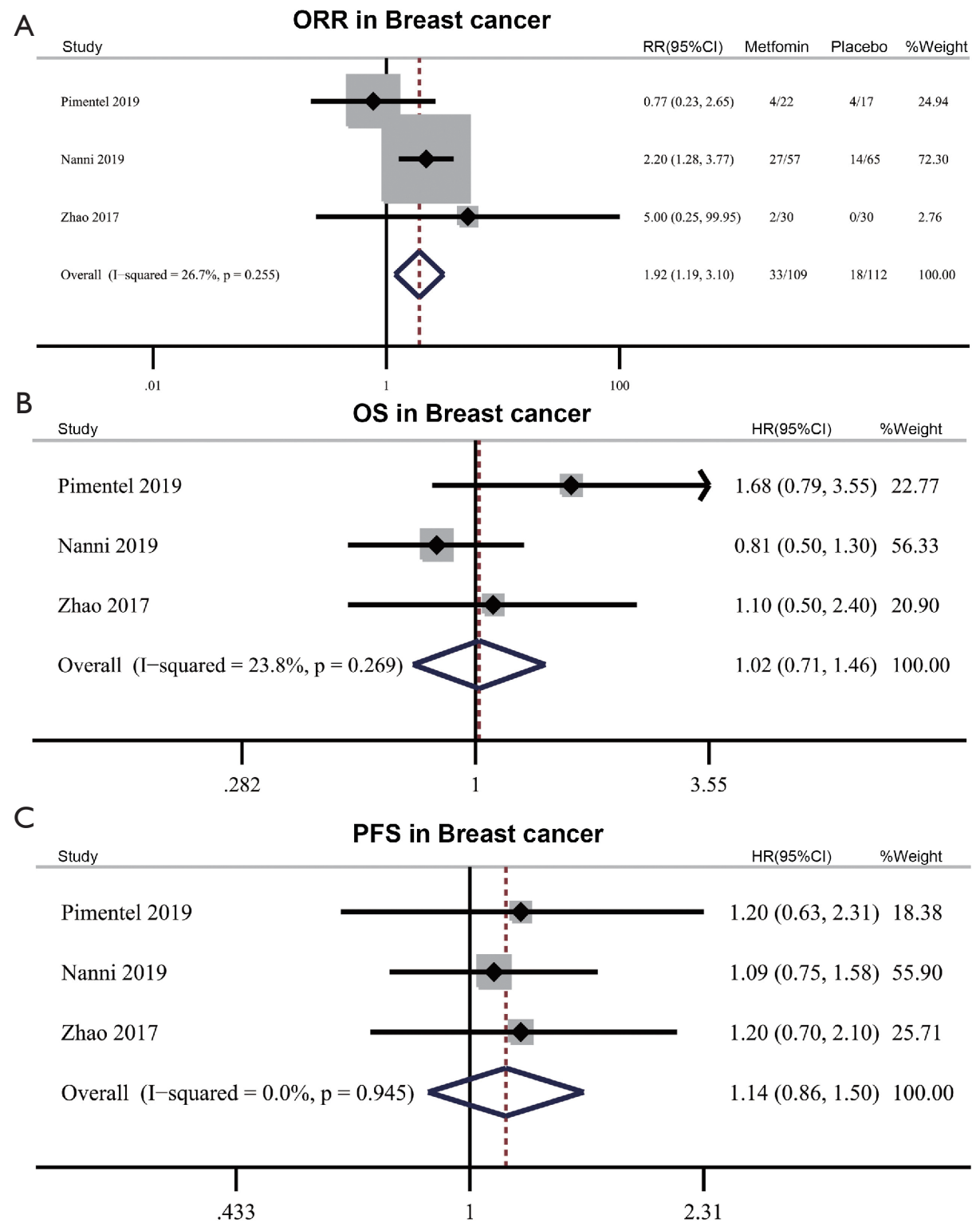

Figure 2 Efficacy of metformin in breast cancer. (A) Effect of metformin plus standard treatment on objective response rate (ORR) in breast cancer. (B,C) Effect of metformin plus standard treatment on overall survival (OS) (B) and progression-free survival (PFS) (C) in breast cancer. $\mathrm{RR}$, relative risk; HR, hazard ratio.

$\mathrm{P}=0.988 ; \mathrm{I}^{2}=0 \%, \mathrm{P}_{\mathrm{het}}=0.522 ; 500 \mathrm{mg}$ daily: HR 0.84, 95\% CI: $0.64-1.15, \mathrm{P}=0.307 ; \mathrm{I}^{2}=41.3 \%, \mathrm{P}_{\text {het }}=0.164$ ) (Figure $\mathrm{S} 1$ ) and PFS (1,000 mg daily: HR 0.90, 95\% CI: 0.53-1.52, $\mathrm{P}=0.686 ; \mathrm{I}^{2}=67.1 \%, \mathrm{P}_{\text {het }}=0.048 ; 500 \mathrm{mg}$ daily: HR 0.93, 95\% CI: 0.59-1.49, $\mathrm{P}=0.775 ; \mathrm{I}^{2}=66.8 \%, \mathrm{P}_{\text {het }}=0.049$ ) (Figure $\mathrm{S} 2$ ).

\section{Publication bias}

Begg's and Egger's tests were performed to evaluate the publication bias. The results of Begg's and Egger's tests showed that no significant publication bias was found in the overall analysis of OS $\left(\mathrm{P}_{\text {Begg's }}=0.602, \mathrm{P}_{\text {egger's }}=0.632\right)$ and PFS $\left(\mathrm{P}_{\text {Begg's }}=0.711, \mathrm{P}_{\text {egger's }}=0.191\right)($ Figure $\mathrm{S} 3)$.

\section{Discussion}

Over the past few decades, accumulating evidence (38) has revealed the anti-cancer effects of metformin. Studies 


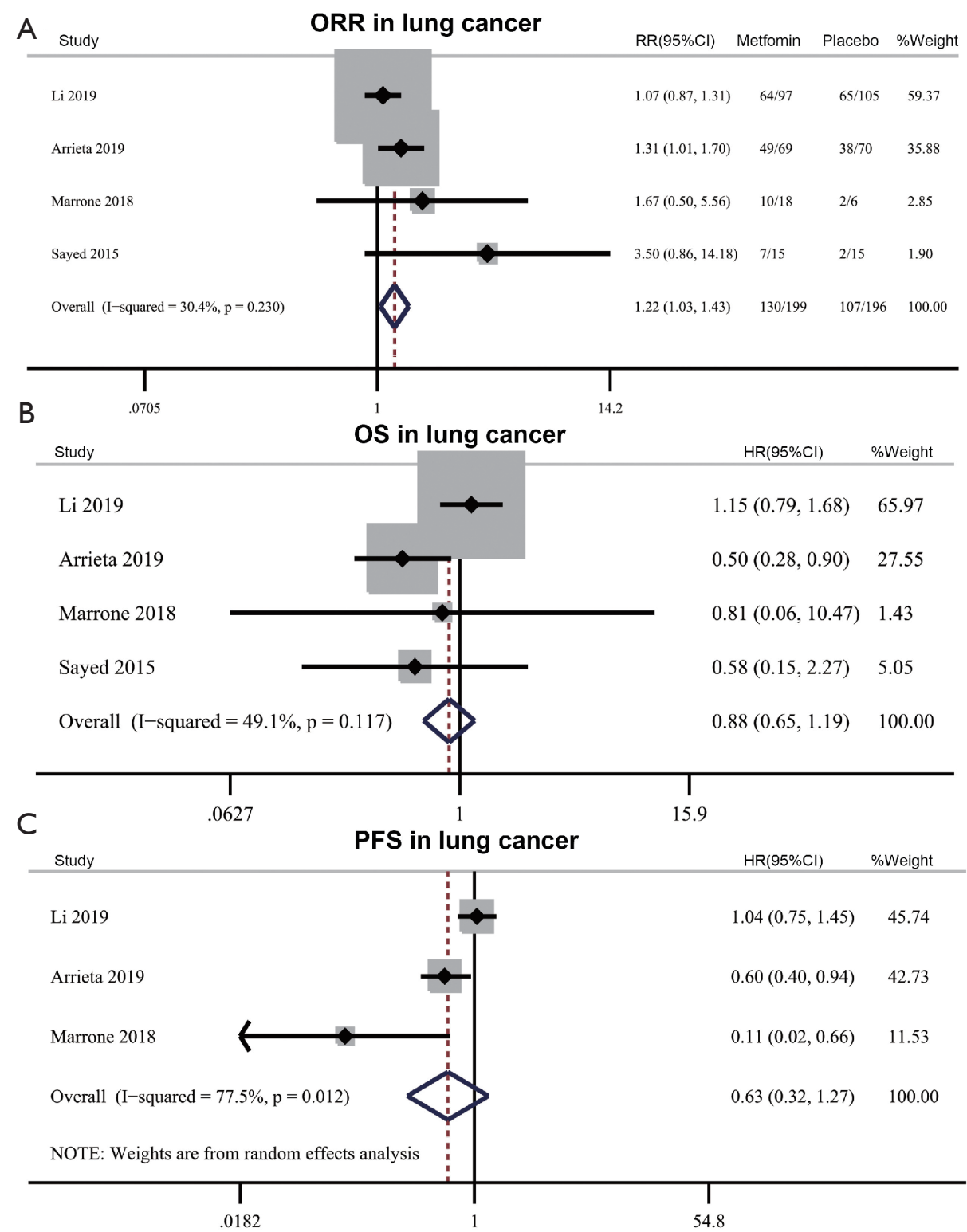

Figure 3 Efficacy of metformin in lung cancer. (A) Effect of metformin plus standard treatment on objective response rate (ORR) in lung cancer. (B,C) Effect of metformin plus standard treatment on overall survival (OS) (B) and progression-free survival (PFS) (C) in lung cancer. $\mathrm{RR}$, relative risk; $\mathrm{HR}$, hazard ratio.

have reported that metformin can reduce the risks of pancreatic, prostate and lung cancer, even after adjusting for confounding factors such as age, smoking status and glycated hemoglobin levels. In recent years, the benefits of metformin in the survival outcomes of patients with breast, prostate, colorectal, endometrial and lung cancer have been documented in several retrospective studies $(39,40)$. Recently, prospective studies have investigated the efficacy of adding metformin to standard treatment for cancer patients, yet a consensus is yet to be reached. Thus, our meta-analysis of current studies provides a greater understanding of the benefits of adding metformin to standard treatment in cancer patients.

To our knowledge, this is the first meta-analysis that systematically evaluated the efficacy and safety profiles on addition of metformin to standard treatment for cancer patients. Our results indicated that metformin combined with standard treatment could improve the ORR compared 
A

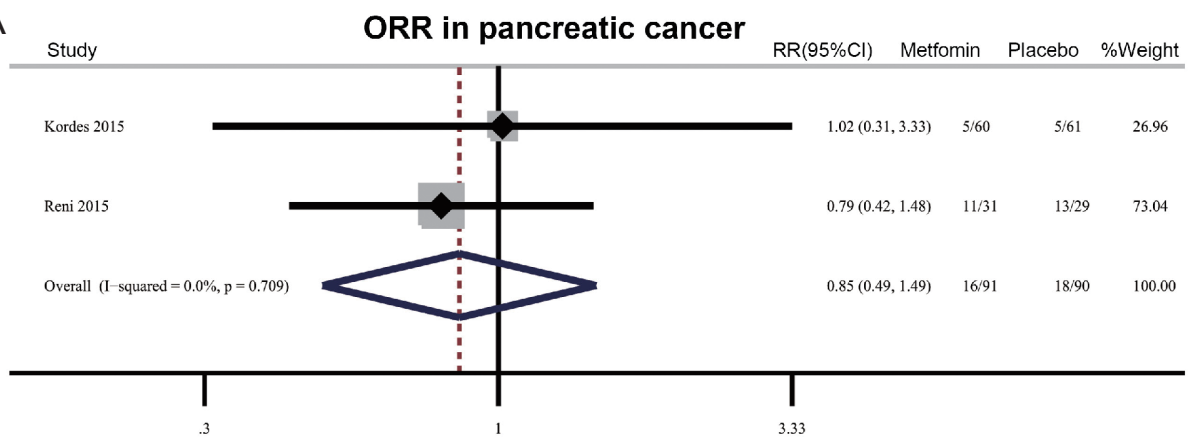

B

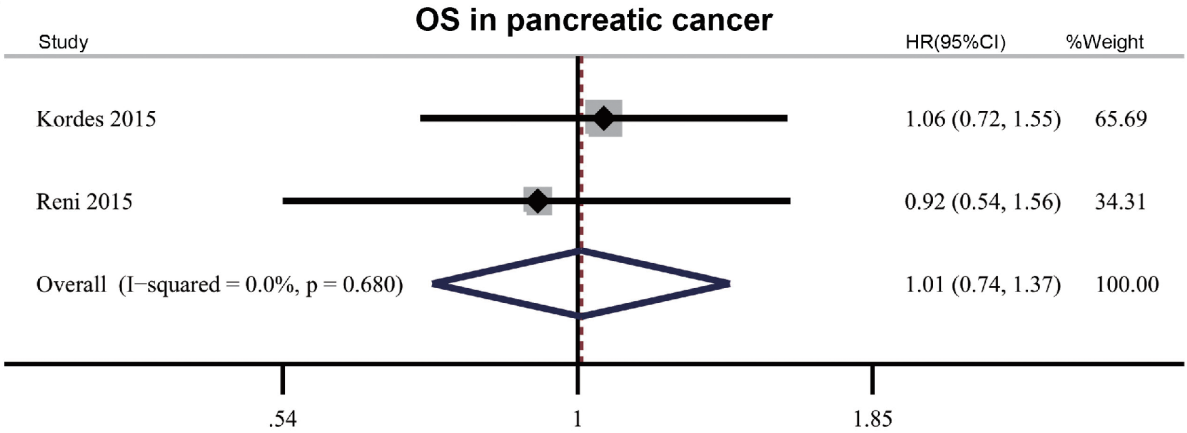

C

PFS in pancreatic cancer

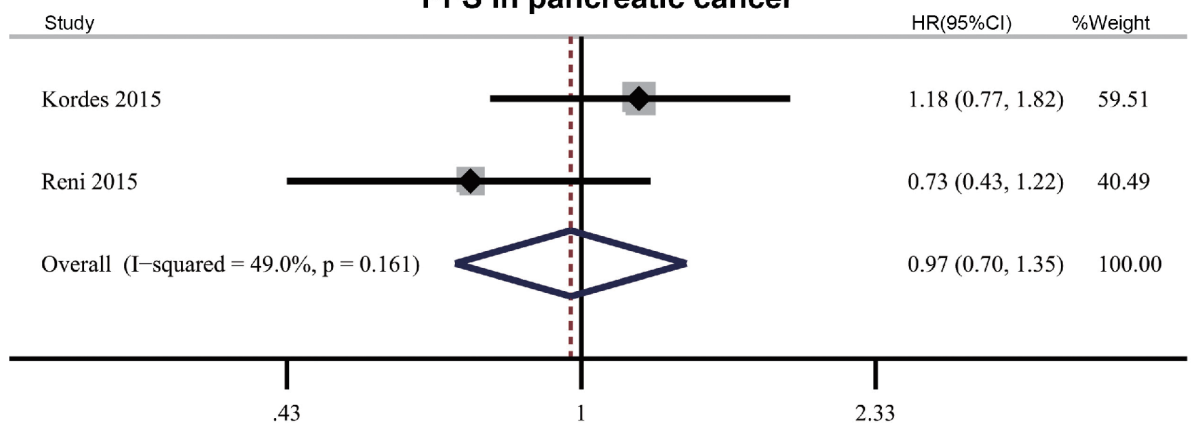

Figure 4 Efficacy of metformin in pancreatic cancer. (A) Effect of metformin plus standard treatment on objective response rate (ORR) in pancreatic cancer. (B,C) Effect of metformin plus standard treatment on overall survival (OS) (B) and progression-free survival (PFS) (C) in pancreatic cancer. RR, relative risk; HR, hazard ratio.

with receiving standard treatment alone in inoperable cancer patients. The analysis of the incidence of AEs indicated that the addition of metformin to standard treatment did not increase the risk of AEs, except for a moderate increase in the incidence of diarrhea in the metformin plus standard treatment group. However, the addition of metformin to standard treatment did not improve OS or PFS among inoperable cancer patients compared with standard treatment alone, indicating that metformin did not bring survival benefit to inoperable cancer patients.

Subgroup analysis based on the metformin dose also showed both $500 \mathrm{mg}$ metformin and 1,000 $\mathrm{mg}$ metformin daily could benefit the ORR. These results indicated that these metformin doses applied in these included studies are suitable for cancer patients and are worth investigating further. From the results, we showed that the $500 \mathrm{mg}$ provided a better effect compared with $1,000 \mathrm{mg}$. As was known to us, metformin plays an anti-cancer effect mainly through acting on AMPK/mTOR pathway. A previous study showed metformin inhibited mTOR signaling via a dose-dependent mechanism, revealing that low-dose metformin directly inhibited mTOR through AMPK and TSC pathway, while high-dose metformin may work through other ways (41). The findings of this study may 
Table 2 Meta-analysis of toxicities in cancer patients receiving metformin plus standard treatment

\begin{tabular}{|c|c|c|c|c|c|c|}
\hline Toxicities & $\mathrm{N}$ & Metformin & Placebo & $\mathrm{RR}(95 \% \mathrm{Cl})$ & $P$ value & $\begin{array}{l}\text { Heterogeneity } \\
\qquad\left(I^{2}, P \text { value }\right)\end{array}$ \\
\hline Neutropenia & 4 & $56 / 165$ & $64 / 162$ & $0.86(0.66-1.12)$ & 0.258 & $14 \%, 0.315$ \\
\hline Febrile neutropenia & 2 & $2 / 75$ & $7 / 71$ & $0.22(0.04-1.17)$ & 0.076 & $0 \%, 0.734$ \\
\hline Anemia & 3 & $2 / 102$ & $3 / 110$ & $0.79(0.18-3.49)$ & 0.758 & $0 \%, 0.592$ \\
\hline Nausea & 4 & $7 / 234$ & $4 / 232$ & $1.52(0.48-4.85)$ & 0.479 & $0 \%, 0.900$ \\
\hline Vomiting & 6 & $9 / 345$ & $5 / 343$ & $1.363(0.501-3.706)$ & 0.544 & $0 \%, 0.609$ \\
\hline Fatigue & 4 & $10 / 216$ & $7 / 226$ & $1.50(0.60-3.75)$ & 0.386 & $0 \%, 0.407$ \\
\hline Diarrhea & 5 & $26 / 327$ & $14 / 337$ & $1.871(1.002-3.494)$ & 0.049 & $0 \%, 0.929$ \\
\hline Alopecia & 3 & $1 / 147$ & $3 / 156$ & $0.46(0.07-3.01)$ & 0.417 & $0 \%, 0.403$ \\
\hline Rash & 2 & $8 / 171$ & $12 / 172$ & $0.67(0.28-1.60)$ & 0.369 & $0 \%, 0.985$ \\
\hline
\end{tabular}

$\mathrm{N}$, number of included studies; RR, relative risk; Cl: confidence interval.

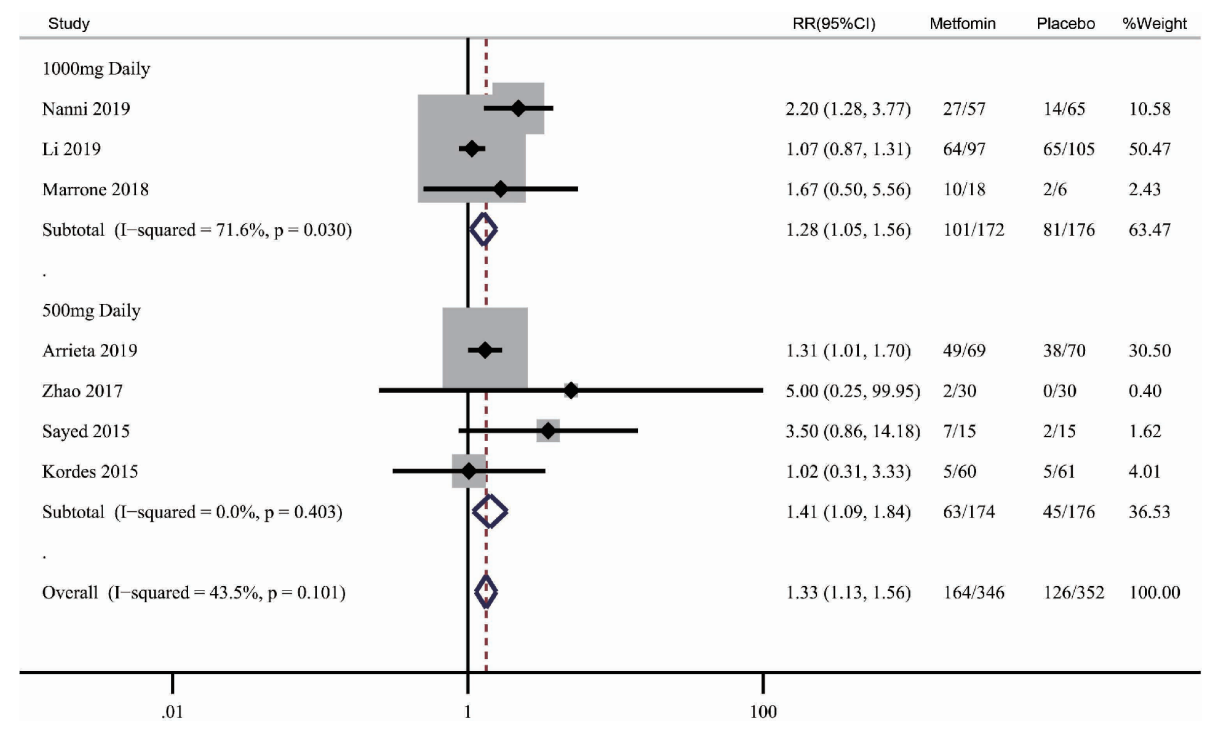

Figure 5 Subgroup analysis of metformin plus standard treatment on objective response rate (ORR) based on metformin doses. RR, relative risk.

partially explain why low metformin dose provided better effect. Additionally, no significantly increased incidence of common AEs was observed after addition of metformin to standard treatment, showing the metformin dose was relatively safe for advanced or metastatic cancer patients. Subgroup analysis based on the cancer type revealed that addition of metformin to standard treatment was beneficial to ORR in breast cancer and lung cancer, whereas metformin did not show a significant benefit for pancreatic cancer patients. These findings may be explained by the fact that the pancreas is an endocrine gland that can secrete various kinds of hormones, including insulin and glucagon 
to modulate the body's metabolism. The pancreatic cancer cells originating from the pancreas may still reserve the secretory function, and often such function is dysregulated in this pathogenic state and this may interfere with the function of metformin in regulating the metabolism.

The anti-cancer effects of metformin may be beneficial in other situations as well, for instance, in early-stage cancer or adjuvant therapy settings. A previously published cohort study of type II diabetic patients with NSCLC in the United States' military health system has reported survival benefits for early-stage patients receiving metformin (42). Moreover, a recent meta-analysis (4) of 24,178 participants from 27 eligible studies has revealed that metformin is a useful adjuvant, with survival benefits in patients with earlystage colorectal and prostate cancer. Collectively, these findings indicate that further trials investigating the benefits of adding metformin to neoadjuvant or adjuvant therapy for patients with early-stage or resectable cancer are needed. In addition, there is population-based evidence suggesting that metformin requires long-term use to exert its anti-cancer effect (43). This evidence can partially explain the potential role of metformin in locally advanced cancer patients compared with metastatic cancer patients. Such metastatic cancer patients are usually with shorter survival time and may not be able to receive metformin therapy enough for a therapeutic effect to emerge.

Although adding metformin to standard treatment did not bring survival benefit to inoperable cancer patients, our study is also essential as it raises several points that should be carefully considered. Firstly, no increase in the occurrence of grade $\geq 3 \mathrm{AEs}$ were observed in patients who received metformin combined with standard treatment. Moreover, metformin is well tolerated during clinical application and the risk of hypoglycemia is minimal. Secondly, our results show that metformin combined with standard treatment can improve the ORR in cancer patients compared with standard treatment alone, which confirms the efficacy of metformin. Lastly, the negative results of our meta-analysis are invaluable for identifying the precise prognostic or predictive factors in the selection and stratification of patients who are more likely to respond to and benefit from metformin treatment. For instance, studies on oxidative phosphorylation (OXPHOS) in tumors can help to optimize patient selection because metformin has been reported to inhibit mitochondrial OXPHOS (44). Based on these points, future studies evaluating the efficacy of adding metformin to standard treatment regimens should not be abandoned and we hope our result emphasize the validity of this vein of research.

There are several limitations in the current study. Firstly, the efficacy of metformin was assessed in various kinds of cancers, including breast cancer, lung cancer, pancreatic cancer, which inevitably increased the heterogeneity among these studies, although low heterogeneity was noted in the analysis. For some overall and subgroup analysis, the number of included studies was somewhat small, which may influence the statistical power of analysis to some degree. Secondly, the study design of some included trials were prospective single-institution RCTs with small sample sizes, which might have reported small-study effects, thereby over-emphasizing the final results. This meta-analysis relied on published data from eligible studies, and we could not further obtain individual patient data that might have influenced the efficacy of metformin. Thirdly, our metaanalysis was not registered in a publicly available repository. However, our meta-analysis is performed strictly in accordance with the process of systematic review and metaanalysis.

\section{Conclusions}

In summary, the results of our meta-analysis indicate that adding metformin to standard treatment can improve the ORR in cancer patients compared with standard treatment alone. Moreover, the addition of metformin to standard treatment does not increase the risk of AEs. However, adding metformin to standard treatment did not benefit OS and PFS in inoperable cancer patients.

\section{Acknowledgments}

Funding: This work was supported by Major R\&D Plan of Liaoning Province [2019020176-JH1/103], General Program of China Postdoctoral Science Foundation [2018M640267], Special funding of China Postdoctoral Science Foundation [2019T120223] and Support plan for young and middle-aged scientific and technological talents in Shenyang [RC190202]. The Natural Science Foundation Medical and Health Joint Fund Project of Liaoning Province (20180530006).

\section{Footnotes}

Reporting Checklist: The authors have completed the PRISMA reporting Checklist. Available at http://dx.doi. org/10.21037/atm-20-4441 
Peer Review File: Available at http://dx.doi.org/10.21037/ atm-20-4441

Conflicts of Interest: All authors have completed the ICMJE uniform disclosure form (available at http://dx.doi. org/10.21037/atm-20-4441). The authors have no conflicts of interest to declare.

Ethical Statement: The authors are accountable for all aspects of the work in ensuring that questions related to the accuracy or integrity of any part of the work are appropriately investigated and resolved. This study was approved by the institutional Ethics Committee of the First Affiliated Hospital of China Medical University (IRB approval number:AF-SOP-07-1.1-01). The informed consent was waived because this study was a meta-analysis of previously published RCTs and did not involve any processing of individual patient data.

Open Access Statement: This is an Open Access article distributed in accordance with the Creative Commons Attribution-NonCommercial-NoDerivs 4.0 International License (CC BY-NC-ND 4.0), which permits the noncommercial replication and distribution of the article with the strict proviso that no changes or edits are made and the original work is properly cited (including links to both the formal publication through the relevant DOI and the license). See: https://creativecommons.org/licenses/by-nc-nd/4.0/.

\section{References}

1. Bray F, Ferlay J, Soerjomataram I, et al. Global cancer statistics 2018: GLOBOCAN estimates of incidence and mortality worldwide for 36 cancers in 185 countries. CA Cancer J Clin 2018;68:394-424.

2. GBD 2017 Causes of Death Collaborators. Global, regional, and national age-sex-specific mortality for 282 causes of death in 195 countries and territories, 1980-2017: a systematic analysis for the Global Burden of Disease Study 2017. Lancet 2018;392:1736-88.

3. Amin S, Mhango G, Lin J, et al. Metformin Improves Survival in Patients with Pancreatic Ductal Adenocarcinoma and Pre-Existing Diabetes: A Propensity Score Analysis. Am J Gastroenterol 2016;111:1350-7.

4. Coyle C, Cafferty FH, Vale C, et al. Metformin as an adjuvant treatment for cancer: a systematic review and meta-analysis. Ann Oncol 2016;27:2184-95.
5. Cardwell CR, Hicks BM, Hughes C, et al. Statin use after colorectal cancer diagnosis and survival: a populationbased cohort study. J Clin Oncol 2014;32:3177-83.

6. Gopalan A, Yu W, Sanders BG, et al. Simvastatin inhibition of mevalonate pathway induces apoptosis in human breast cancer cells via activation of JNK/CHOP/ DR5 signaling pathway. Cancer Lett 2013;329:9-16.

7. Li L, Han R, Xiao H, et al. Metformin sensitizes EGFRTKI-resistant human lung cancer cells in vitro and in vivo through inhibition of IL-6 signaling and EMT reversal. Clin Cancer Res 2014;20:2714-26.

8. Morgillo F, Sasso FC, Della Corte CM, et al. Synergistic effects of metformin treatment in combination with gefitinib, a selective EGFR tyrosine kinase inhibitor, in LKB1 wild-type NSCLC cell lines. Clin Cancer Res 2013;19:3508-19.

9. Wang F, Liu W, Ning J, et al. Simvastatin Suppresses Proliferation and Migration in Non-small Cell Lung Cancer via Pyroptosis. Int J Biol Sci 2018;14:406-17.

10. Ben Sahra I, Le Marchand-Brustel Y, Tanti JF, et al. Metformin in cancer therapy: a new perspective for an old antidiabetic drug? Mol Cancer Ther 2010;9:1092-9.

11. Kisfalvi K, Eibl G, Sinnett-Smith J, et al. Metformin disrupts crosstalk between $\mathrm{G}$ protein-coupled receptor and insulin receptor signaling systems and inhibits pancreatic cancer growth. Cancer Res 2009;69:6539-45.

12. Courtois S, Lehours P, Bessede E. The therapeutic potential of metformin in gastric cancer. Gastric Cancer 2019;22:653-62.

13. Wang QL, Santoni G, Ness-Jensen E, et al. Association Between Metformin Use and Risk of Esophageal Squamous Cell Carcinoma in a Population-Based Cohort Study. Am J Gastroenterol 2020;115:73-8.

14. Lohmann AE, Pimentel I, Goodwin PJ. Novel Insights Into the Impact of Lifestyle-Based Weight Loss and Metformin on Obesity-Associated Biomarkers in Breast Cancer. J Natl Cancer Inst 2018;110:1161-2.

15. Xiao K, Liu F, Liu J, et al. The effect of metformin on lung cancer risk and survival in patients with type 2 diabetes mellitus: A meta-analysis. J Clin Pharm Ther 2020;45:783-92.

16. Yao L, Liu M, Huang Y, et al. Metformin Use and Lung Cancer Risk in Diabetic Patients: A Systematic Review and Meta-Analysis. Dis Markers 2019;2019:6230162.

17. Wirunsawanya K, Jaruvongvanich V, Upala S. Survival Benefits From Metformin Use in Pancreatic Cancer: A Systemic Review and Meta-analysis. Pancreas 
2018;47:e11-4.

18. Wan G, Sun X, Li F, et al. Survival Benefit of Metformin Adjuvant Treatment For Pancreatic Cancer Patients: a Systematic Review and Meta-Analysis. Cell Physiol Biochem 2018;49:837-47.

19. Jian-Yu E, Graber JM, Lu SE, et al. Effect of Metformin and Statin Use on Survival in Pancreatic Cancer Patients: a Systematic Literature Review and Meta-analysis. Curr Med Chem 2018;25:2595-607.

20. Lin JJ, Gallagher EJ, Sigel K, et al. Survival of patients with stage IV lung cancer with diabetes treated with metformin. Am J Respir Crit Care Med 2015;191:448-54.

21. Zeng S, Gan HX, Xu JX, et al. Metformin improves survival in lung cancer patients with type 2 diabetes mellitus: A meta-analysis. Med Clin (Barc) 2019;152:291-7.

22. Xu H, Chen K, Jia X, et al. Metformin Use Is Associated With Better Survival of Breast Cancer Patients With Diabetes: A Meta-Analysis. Oncologist 2015;20:1236-44.

23. Yang T, Yang Y, Liu S. Association between Metformin Therapy and Breast Cancer Incidence and Mortality: Evidence from a Meta-Analysis. J Breast Cancer 2015;18:264-70.

24. Moher D, Cook DJ, Eastwood S, et al. Improving the quality of reports of meta-analyses of randomised controlled trials: the QUOROM statement. Quality of Reporting of Meta-analyses. Lancet 1999;354:1896-900.

25. Higgins JP, Thompson SG, Deeks JJ, et al. Measuring inconsistency in meta-analyses. BMJ 2003;327:557-60.

26. Egger M, Davey Smith G, Schneider M, et al. Bias in meta-analysis detected by a simple, graphical test. BMJ 1997;315:629-34.

27. Begg CB, Mazumdar M. Operating characteristics of a rank correlation test for publication bias. Biometrics 1994;50:1088-101.

28. Sayed R, Saad AS, El Wakeel L, et al. Metformin Addition to Chemotherapy in Stage IV Non-Small Cell Lung Cancer: an Open Label Randomized Controlled Study. Asian Pac J Cancer Prev 2015;16:6621-6.

29. Pimentel I, Lohmann AE, Ennis M, et al. A phase II randomized clinical trial of the effect of metformin versus placebo on progression-free survival in women with metastatic breast cancer receiving standard chemotherapy. Breast 2019;48:17-23.

30. Nanni O, Amadori D, De Censi A, et al. Metformin plus chemotherapy versus chemotherapy alone in the first-line treatment of HER2-negative metastatic breast cancer. The MYME randomized, phase 2 clinical trial. Breast Cancer Res Treat 2019;174:433-42.
31. Li L, Jiang L, Wang Y, et al. Combination of Metformin and Gefitinib as First-Line Therapy for Nondiabetic Advanced NSCLC Patients with EGFR Mutations: A Randomized, Double-Blind Phase II Trial. Clin Cancer Res 2019;25:6967-75.

32. Reni M, Dugnani E, Cereda S, et al. (Ir)relevance of Metformin Treatment in Patients with Metastatic Pancreatic Cancer: An Open-Label, Randomized Phase II Trial. Clin Cancer Res 2016;22:1076-85.

33. Arrieta O, Barron F, Padilla MS, et al. Effect of Metformin Plus Tyrosine Kinase Inhibitors Compared With Tyrosine Kinase Inhibitors Alone in Patients With Epidermal Growth Factor Receptor-Mutated Lung Adenocarcinoma: A Phase 2 Randomized Clinical Trial. JAMA Oncol 2019;5:e192553.

34. Kordes S, Pollak MN, Zwinderman AH, et al. Metformin in patients with advanced pancreatic cancer: a doubleblind, randomised, placebo-controlled phase 2 trial. Lancet Oncol 2015;16:839-47.

35. Marrone KA, Zhou X, Forde PM, et al. A Randomized Phase II Study of Metformin plus Paclitaxel/Carboplatin/ Bevacizumab in Patients with Chemotherapy-Naive Advanced or Metastatic Nonsquamous Non-Small Cell Lung Cancer. Oncologist 2018;23:859-65.

36. Zhao Y, Gong C, Wang Z, et al. A randomized phase II study of aromatase inhibitors plus metformin in pre-treated postmenopausal patients with hormone receptor positive metastatic breast cancer. Oncotarget 2017;8:84224-36.

37. Kim J, Han W, Kim EK, et al. Phase II randomized study of neoadjuvant metformin plus letrozole versus placebo plus letrozole for ER-positive postmenopausal breast cancer [METEOR Study]. J Clin Oncol 2019;37:576.

38. Dowling RJ, Goodwin PJ, Stambolic V. Understanding the benefit of metformin use in cancer treatment. BMC Med 2011;9:33.

39. Chen H, Yao W, Chu Q, et al. Synergistic effects of metformin in combination with EGFR-TKI in the treatment of patients with advanced non-small cell lung cancer and type 2 diabetes. Cancer Lett 2015;369:97-102.

40. Currie CJ, Poole CD, Jenkins-Jones S, et al. Mortality after incident cancer in people with and without type 2 diabetes: impact of metformin on survival. Diabetes Care 2012;35:299-304.

41. Howell JJ, Hellberg K, Turner M, et al. Metformin Inhibits Hepatic mTORC1 Signaling via Dose-Dependent Mechanisms Involving AMPK and the TSC Complex. Cell Metab 2017;25:463-71.

42. Lin J, Gill A, Zahm SH, et al. Metformin use and survival 
after non-small cell lung cancer: A cohort study in the US Military health system. Int J Cancer 2017;141:254-63.

43. Bodmer M, Meier C, Krähenbühl S, et al. Long-term metformin use is associated with decreased risk of breast cancer. Diabetes Care 2010;33:1304-8.

44. Molina JR, Sun Y, Protopopova M, et al. An inhibitor of oxidative phosphorylation exploits cancer vulnerability. Nat Med 2018;24:1036-46.

Cite this article as: $\mathrm{Wu} \mathrm{Z,} \mathrm{Qu} \mathrm{B,} \mathrm{Huang} \mathrm{X,} \mathrm{Song} \mathrm{Y,} \mathrm{Gao} \mathrm{P,}$ Shi J, Zhou C, Wang Z. The potential adjunctive benefit of adding metformin to standard treatment in inoperable cancer patients: a meta-analysis of randomized controlled trials. Ann Transl Med 2020;8(21):1404. doi: 10.21037/atm-20-4441 